\title{
Patterns of reflex excitability change after widespread cutaneous stimulation in $\operatorname{man}^{1}$
}

\author{
M. M. GASSEL AND K. H. OTT \\ From the Department of Neurology, University of California School of Medicine, \\ San Francisco, California, U.S.A.
}

SUMMARY A single-shock stimulus to the skin of widespread and distant parts of the body such as the face, sites in the upper limb, trunk, buttock, and feet produced changes in amplitude of the $\vec{O}$ ankle jerk. A regulated and stabilized system was used for eliciting the ankle jerk and for delivering an unvarying single-shock conditioning stimulus; 35 normal subjects were studied. The characteristics of the recovery curve of the monosynaptic reflex after stimulation at these cutaneous sites are described.

The reflex movements evoked by variously located cutaneous stimuli were studied by Sherrington (1906) in spinal and decerebrate animals. He distinguished short spinal reflexes, with muscular responses within the same locality as that of the application of the stimulus, from long spinal reflexes evoked at a distance. The long spinal reflexes involved neck, trunk, and interlimb postural adjustments which appeared to irradiate from the local site of application of the stimulus and were of the type represented in the 'reflex figure'. Lloyd (1942) observed facilitation of monosynaptic reflexes in the lower extremity from 7 to $35 \mathrm{msec}$ after conditioning with single-shocks to the brachial plexus in cats; inhibition at 3 to 6 msec sometimes preceded the facilitation. The shortest latency of the onset of excitability change indicated a direct descending influence with interposition of but one synapse. Kugelberg, Eklund, and Grimby (1960) concluded that the nociceptive reflexes of the lower limb in man are coordinated movements which may involve the entire ipsilateral limb, lower part of the trunk, and the proximal part of the contralateral limb. Gernandt and Shimamura (1961) and Shimamura and colleagues (Shimamura and Livingston, 1963; Shimamura and Akert, 1965) have distinguished two longitudin-

1 This work was supported in part by NIH grant NB-07562 (Dr. Gassel) and USPHS Fellowships FR-05355-07 and NB-5101-13 (Dr. Ott). ally oriented systems concerned with reflex $\stackrel{\infty}{N}_{\infty}$ activity, which can be activated by stimulation of 0 dorsal roots at any level. One system is desig nated 'the propriospinal system' and consists of $>$ slowly and directly conducting ascending or deㅡㄹ scending spinal pathways with multiple crossings $\vec{\theta}$ of the spinal cord. A long-loop, spino-bulb spinal, or trigeminal-bulbo-spinal system is 0 more rapidly conducting, especially on the afferent side, and ascends to the bulbar reticular formation before descending and producing serial and bilateral ventral root discharges. Long-loop reflexes have been reported to involve $\overline{\vec{\partial}}$ only the flexor muscles, with stimulation of skin 3 afferents (Shimamura and Akert, 1965). There is an overlap of reflexes evoked by the propriospinal and spino-bulbo-spinal systems in the forelimb on stimulation of hindlimb afferents. This results from the discrepancy in conduction velocities, with the slower conducting direct propriospinal reflexes arriving at the same time as the more rapidly conducting spino-bulbospinal reflexes. A similar coincidence occurs in the forelimb when stimulating hindlimb afferents. This coincidence is thought to provide a bulbar integrating effect on interlimb reflexes.

This study reports the excitability changes in a $N$ population of triceps surae motoneurones on $\underset{\omega}{N}$ stimulation of widespread cutaneous areas in $\vec{Z}$ humans. A regulated system is used for eliciting 6 the test monosynaptic reflex and for delivering a 
cutaneous conditioning stimulus which is unvarying (Gassel and Ott, 1970). Excitability changes are evaluated by the monosynaptic reflex recovery curve with classical conditioning techniques.

It will be shown that significant and prolonged effects on excitability of the triceps surae motoneurones are produced by single shock stimulation of distant cutaneous areas, such as the face, arm, back, or contralateral foot. The pattern and range of excitability changes on stimulation of specific skin areas in a group of normal subjects are described.

\section{METHODS}

The subjects were placed in a prone position with legs extending over the end of the couch. The leg was supported firmly in a metal frame which was attached to a metal stand separated from the couch. An automatic solenoid hammer was fixed to the leg frame by a metal supporting device with two complex joints which made it possible to position the hammer over the Achilles tendon (Gassel and Ott, 1970). The solenoid was triggered from the stimulator (DISA Multistim) and the delay to the tendon tap was about $55 \mathrm{msec}$. The delay was measured at each experiment. The solenoid driven reflex hammer had a built-in microswitch which closed the circuit on percussion and triggered the sweep of the electromyograph. A regular and stabilized blow was delivered. The evoked muscle action potential was recorded with non-insulated needle electrodes, one placed over the belly and one over the tendon of the triceps surae muscle. The potentials were amplified and displayed on the DISA 3-channel electromyograph. The recovery curve of the ankle jerk reflex after singlechock cutaneous stimulation was plotted. A DISA Multistim stimulator was used with a maximum voltage output, via transformer, of $250 \mathrm{~V}$. Single $3 \mathrm{msec}$ duration square-wave pulses were delivered with subcutaneous stainless steel electrodes placed $2 \mathrm{~cm}$ apart. A conditioning shock, painful but of tolerable intensity, was delivered to the distal contralateral foot, dorsal and plantar surfaces, ipsilateral or contralateral shoulder, ipsilateral or contralateral arm, ipsilateral hand, ipsilateral face, back, or buttock. The shock was followed at intervals ranging from 0 to $8,000 \mathrm{msec}$ by the ankle jerk reflex. Maximal monosynaptic reflexes were elicited at a frequency of one every $10 \mathrm{sec}$. The amplitudes of eight reflexes were averaged by computer (CAT 1000 ) in establishing the control and test values; in some studies 20 reflexes were averaged when control variations were large. The controls were repeated at intervals during the investigation and the test values normalized to control.

Thirty-five normal subjects were evaluated. The subjects were paid volunteers and laboratory staff with the capacity to remain relaxed for long periods in the prone position. Each recovery curve took about two hours to plot. A total of 90 recovery curves were studied. These included 27 studies of the contralateral foot, 20 of the shoulder (15 ipsilateral, 5 contralateral), 15 buttock, 10 arm ( 6 ipsilateral, 4 contralateral), and 6 face. One subject C.J., was a particularly tolerant medical colleague, and a total of 25 separate recovery curves were plotted over a two and a half year period.

\section{RESULTS}

The changes in excitability evoked in a population of triceps surae motoneurones by a single shock stimulus to each of widespread sites of the cutaneous surface of the body of C.J. are illustrated in Fig. 1. The excitability changes were evaluated by the recovery curve of the monosynaptic reflex (ankle jerk). Recovery curves with stimuli to the dorsal and plantar surfaces of the distal ipsilateral foot have been reported (Gassel and Ott, 1970). Representative curves with variously located conditioning stimuli from four normal subjects are illustrated in Fig. 2, demonstrating the range of normal variation. There was considerable reliability in the pattern of excitability change elicited at any location, although minor variations were usual.

The usual onset of excitability change was at $40 \mathrm{msec}$ (20-60 msec). There were generally two phases of facilitation; an early one lasting to about $100 \mathrm{msec}$ followed by a second often accentuated facilitation with a peak at 100 $200 \mathrm{msec}$. A mild inhibition sometimes followed with gradual or irregular return of reflex excitability to control levels in 1 to $8 \mathrm{sec}$.

PARTICULAR PATTERNS OF EXCITABILITY CHANGE Foot: (1) ipsilateral. There were early excitability changes with 'local sign' at 40-90 msec. Facilitation occurred with stimulation of the dorsal surface and inhibition on plantar stimulation. A prominent late increase of excitability occurred at 110-250 msec with peak at about 150 msec. (2) contralateral. Facilitation usually began at $40 \mathrm{msec}$ and persisted until $200 \mathrm{msec}$. A mild accentuation of the facilitation occasionally 

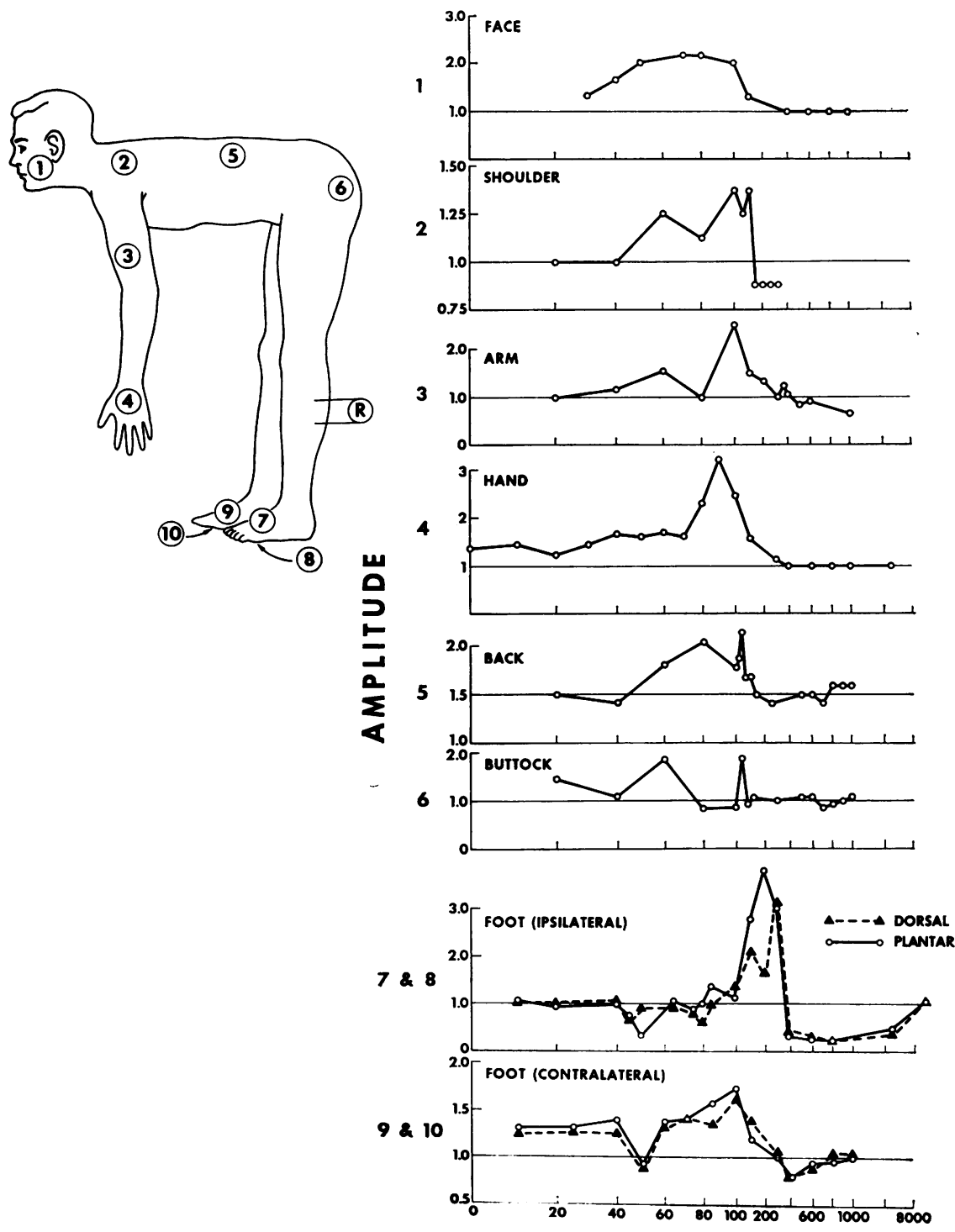

MSEC

FIG. 1. Recovery curves, numbered 1-10, of the monosynaptic reflex (left ankle jerk) conditioned by a single shock stimulus to each of 10 cutaneous sites in subject C.J. The large numerals (1 to 10) on the graphs on the right correspond to the numbered sites on the Figure of the homunculus on the left. Each graphic point represents the average of amplitudes from 10 to 20 reflexes, normalized to control. Amplitude is indicated on the ordinate and the interval in msec between conditioning shock and test reflex on the abscissa. 


\section{$\left\{\begin{array}{c}5 \\ \text { ล }\end{array}\right.$}

(2)

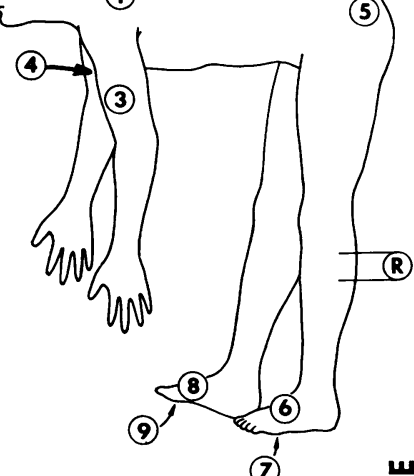

(R)

1

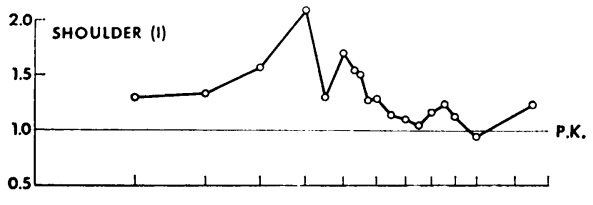

2

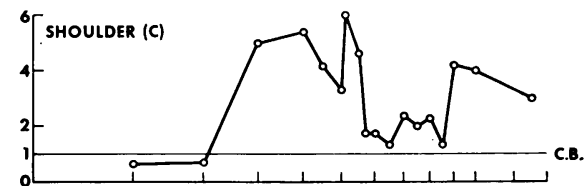

3
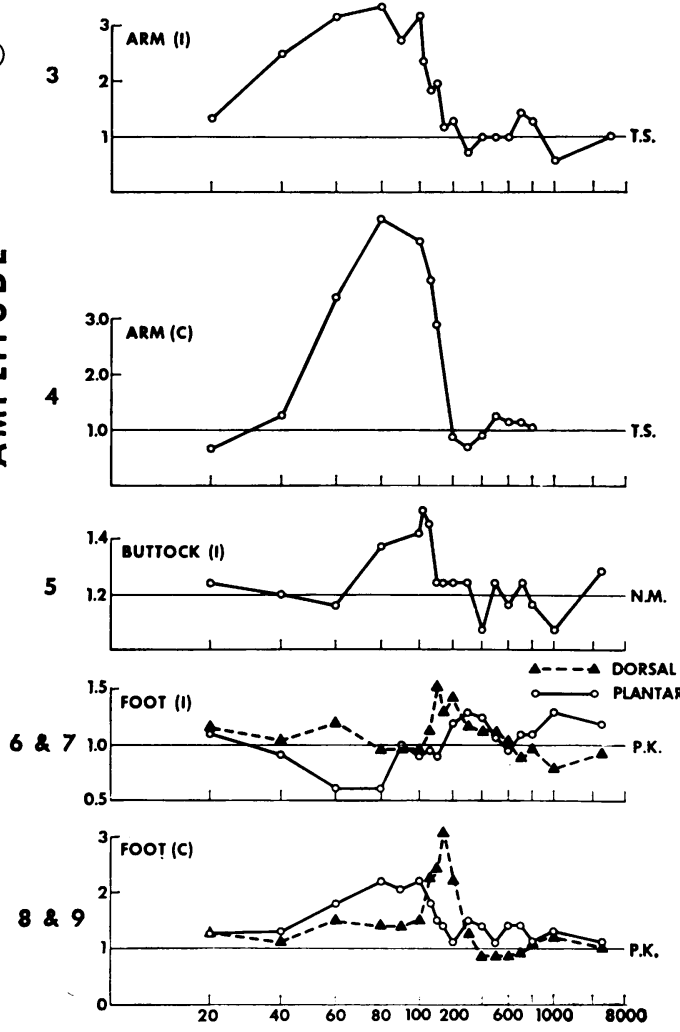

MSEC

FIG. 2. Representative recovery curves of the monosynaptic reflex (ankle jerk) conditioned by a single-shock stimulus to the cutaneous sites indicated, from four normal subjects. The large numerals on the graphs on the right correspond to the numbered sites on the Figure of the homunculus on the left. Each graphic point represents the average of amplitudes from 8 to 20 reflexes, normalized to control. Amplitude is indicated on the ordinate and the interval in msec between conditioning shock and test reflex on the abscissa. 
occurred at $150 \mathrm{msec}$. The recovery curves with dorsal and plantar stimuli were similar and scmetimes almost identical (Fig. 1). Infrequently a mild inhibition preceded the facilitation at $40 \mathrm{msec}$. The usual recovery curve is represented in Fig. 2.

Buttock and back There was onset of early facilitation at about $40 \mathrm{msec}$. A second phase of facilitation had a peak at about $100 \mathrm{msec}$, and sometimes the peak had a sharp rise.

Shoulder and arm The onset of facilitation was at $20-40 \mathrm{msec}$ and it persisted until 100-150 msec. The late phase was often indistinct and occurred at about $80 \mathrm{msec}$.

Hand The onset of change was poorly defined but usually occurred about $40 \mathrm{msec}$. There was sometimes increased facilitation at $80-100 \mathrm{msec}$.

Face There was a single phase of facilitation beginning at $20-40 \mathrm{msec}$ and lasting until 200 msec.

\section{DISCUSSION}

There are typically two phases of facilitation of excitability of the monosynaptic reflex evoked by single-shock stimulation over any of widespread cutaneous sites. The phases are distinct on stimulation of the foot and tend to overlap with stimuli to the back and shoulder. We have previously found that stimuli applied to the dorsal or plantar surface of the ipsilateral foot evoked early excitability changes in the triceps surae muscle with local sign; facilitation on stimulation of the dorsal surface, and inhibition on stimulation of the sole of the ipsilateral foot. The early excitability changes were judged, from the latency of effect, to be mediated by group II cutaneous nerve fibres. A late facilitation without local sign was concluded to be mediated by the smallest skin afferent fibres with a possible contribution from long-loop, spino-bulbo-spinal influences (Gassel and Ott, 1970).

Stimulation of the skin on proximal sites such as the buttock and back, close to the spinal cord reflex centre, decreased the latency of the second facilitation. However, the onset of early change in excitability showed no systematic change with stimuli close (back, shoulder, buttock) or distant (foot, face, hand) to the spinal cord centre. Reflex excitability changes were evaluated in the same population of triceps surae motoneurones, so that the change in latency of the phase of excitability over distance between the sites of stimulation provides a measure of the conduction velocity of the peripheral afferent nerve fibres mediating the facilitation. Utilizing plots with prominent peaks evoked on proximal and distal stimulation in the same individual, it was calculated that the conduction velocity of the late facilitation was of the order of $10-20 \mathrm{~m} / \mathrm{sec}$.

Conclusions about the size of afferent nerve fibres that are based on the change in latency of reflex effects evoked from proximal and distal sites can only be tentative and there are a number of uncertainties. It is, of course, important that the nerve fibres mediating the excitability change at proximal and distal stimulus sites be of the same size. However, there are some differences in the spectrum of nerve fibre diameters from different areas of the skin (Ranson, Droegemueller, Davenport, and Fisher, 온 1935). The largest fibres of cutaneous nerves mediate early changes in excitability with seg mental and crossed spinal reflexes (Hagbart 1952; Perl, 1957; Gassel and Ott, 1970). Yes direct ascending or descending "propriospinal influences mediated by cutaneous nerves at a distance have been reported to be transmitted by high threshold and slowly conducting nerve fibres (Shimamura and Akert, 1965), as well as slower conducting spinal pathways (Shimamura and Livingston, 1963), although this conclusion has not been unanimous (Lloyd, 1942). The precise onset of an excitability change is not always clear. Lloyd showed that the onset of an excitability change might be masked by an interaction between motoneurone fractions with opposite sign of excitability change, but which are tested corporately in the monosynaptic $\delta$ reflex. The onset of effect is not related precisely $₹$ to fibre size as there is a period of "nuclear 음 delay' (Lloyd, 1942), attributable in part to $D$ necessary temporal summation before an influence becomes apparent. It is possible that the $N$ potency of the temporal facilitation from different skin areas on the excitability of the sub- N liminal fringe of triceps surae motoneurones might vary. The range of nerve fibre sizes mediating an excitability change cannot be 
judged directly from the duration of the event, as the cutaneous stimulus induces after-discharge in the skin receptors and a train of nerve volleys.

Possible explanations for the origin of the second facilitation on stimulating cutaneous afferent nerve fibres include long-loop spino-bulbo-spinal influences originally described by Gernandt and Shimamura (1961) or effects of small skin afferent nerve fibres acting directly on the spinal cord reflex centre. The spino-bulbo-spinal system is reported to conduct rapidly with an afferent conduction velocity for spinal pathways of about $60 \mathrm{~m} / \mathrm{sec}$ and an efferent one of $35 \mathrm{~m} / \mathrm{sec}$ in cats (Shimamura and Livingston, 1963). The rate of conduction in the sensory nerve mediating spino-bulbo-spinal reflexes is also high, 50-80 $\mathrm{m} / \mathrm{sec}$ (Shimamura and Akert, 1965). In the present study the conduction velocity of the peripheral afferent nerve fibres engaged in the late excitability change is about $15 \mathrm{~m} / \mathrm{sec}$, consistent with that of small skin afferent nerve fibres rather than the largest cutaneous afferent fibres engaged in spino-bulbo-spinal reflexes. Investigation of patients with complete spinal transections has revealed late excitability changes in triceps surae motoneurones but of reverse sign on stimulation of the skin of the distal foot. This indicates that small afferent fibres can produce effects of this latency with potential longloop influences interrupted, and supports the spinal rather than the long-loop origin of the late facilitation (Gassel and Ott, 1970).

The physiological basis for the late inhibition which is sometimes seen is problematical. It could represent a post-facilitatory effect. Late inhibition of spinal motoneurone excitability has been reported after stimulation of the brachial plexus or sciatic nerve and the inhibition has been interrupted after high spinal section, suggesting the mediation of brain-stem influences in the phenomenon (Gernandt and Shimamura, 1961).

A preliminary study of excitability changes after cutaneous stimulation is being made in patients with selected lesions of the central nervous system and the results have been distinctly abnormal in a number. Distant effects, perhaps exaggerated in stages of ontogeny or in supraspinal lesions, could well provide a neurophysiological basis for such pathological cutaneous reflexes as the palmomental reflex or the distant toe flexor reflex (Wartenberg, 1945).

\section{REFERENCES}

Gassel, M. M., and Ott, K. H. (1970). Local sign and late effects on motoneuron excitability of cutaneous stimulation in man. Brain, 93, 95-106.

Gernandt, B. E., and Shimamura, M. (1961). Mechanisms of interlimb reflexes in cat. Journal of Neurophysiology, 24, 665-676.

Hagbarth, K.-E. (1952). Excitatory and inhibitory skin areas for flexor and extensor motoneurones. Acta Physiologica Scandinavica, 26, Suppl. 94.

Holmqvist, B. (1961). Crossed spinal reflex actions evoked by volleys in somatic afferents. Acta Physiologica Scandinavica, 52, Suppl. 181.

Kugelberg, E., Eklund, K., and Grimby, L. (1960). An electromyographic study of the nociceptive reflexes of the lower limb. Mechanism of the plantar responses. Brain, 83, 394-410.

Lloyd, D. P. C. (1942). Mediation of descending long spinal reflex activity. Journal of Neurophysiology, 5, 435-458.

Perl, E. R. (1957). Crossed reflexes of cutaneous origin. American Journal of Physiology, 188, 609-615.

Ranson, S. W., Droegemueller, W. H., Davenport, H. K., and Fisher, C. (1935). Number, size and myelination of the sensory fibers in the cerebrospinal nerves. Association for Research in Nervous Disease. Proceedings, 15, 3-34.

Sherrington, Sir C. S. (1906). The Integrative Action of the Nervous System. Yale University Press: New Haven.

Shimamura, M., and Akert, K. (1965). Peripheral nervous relations of propriospinal and spino-bulbo-spinal reflex systems. Japanese Journal of Physiology, 15, 638-647.

Shimamura, M., and Livingston, R. B. (1963). Longitudinal conduction systems serving spinal and brain-stem coordination. Journal of Neurophysiology, 26, 258-272.

Wartenberg, R. (1945). The Examination of Reflexes. Year Book Publishers: Chicago. 